\title{
Gestión de inventarios y la rentabilidad de una empresa del sector automotriz
}

Inventory management and profitability of a company in the automotive sector

Gestão de estoques e rentabilidade de uma empresa do setor automotivo

\author{
Magaly Lozano García \\ llozanoga@ucvvirtual.edu.pe \\ Universidad Cesar Vallejo - Perú \\ https://orcid.org/0000-0002-2310-0747 \\ Patsy Fabiola Pezo Dávila \\ ppezoda@ucvvirtual.edu.pe \\ Universidad Cesar Vallejo - Perú \\ https://orcid.org/0000-0002-6713-7242 \\ Segundo Eloy Soto Abanto \\ ssotoa@ucv.edu.pe \\ Universidad Cesar Vallejo - Perú \\ https://orcid.org/0000-0003-1004-5520
}

Avelino Sebastián Villafuerte de la Cruz

avillafuerted@ucvvirtual.edu.pe

Universidad Cesar Vallejo - Perú https://orcid.org/0000-0002-9447-8683

\begin{abstract}
RESUMEN
La investigación tuvo como objetivo Determinar el efecto de la gestión de inventarios en la rentabilidad de la empresa automotriz MOTORS SHOW SAC del distrito de Tarapoto, Provincia y Departamento de San Martin, Perú. Estudio que tuvo presente artículos científicos orientados y publicados en EBSCO HOST, redalyc, ProQuest, SciElo, Scopus, Dialnet y SciencieDirect en base a los últimos 5 años. Metodología: Es de tipo aplicada porque se dependió de aportes teóricos para alternativas de solución, con enfoque cuantitativo, alcance descriptivo, de corte transversal y diseño no experimental porque no se manipularon variables. Como resultado de lo antes mencionado, se evidenció que a inicios del periodo evaluado la gestión de inventarios era de manera deficiente, el cual mejoró de manera progresiva con la aplicación de nuevas políticas generando un efecto positivo en la rentabilidad. La investigación concluye que de acuerdo al análisis realizado la empresa cuenta con herramientas para desarrollar las actividades correctamente hecho que genera mejora en los procesos de gestión y que la implementación de las nuevas políticas ha influido de manera satisfactoria en la rentabilidad generando un ROS de 4.75\%, ROA de 4.48\%, ROE de 37\% Y ROCE de $112 \%$.
\end{abstract}

Palabras clave / Descriptores: Gestión. Inventarios. Políticas. Rentabilidad.

\begin{abstract}
The objective of the research was to determine the effect of inventory management on the profitability of the automotive company in the district of Tarapoto, a study that took into account scientific articles oriented and published in EBSCO HOST, redalyc, ProQuest, SciElo, Scopus, Dialnet and SciencieDirect based on the last 5 years. Methodology: It is applied because it relied on theoretical contributions for solution alternatives, with a quantitative approach, descriptive scope, cross-sectional and non-experimental design because no variables were manipulated. As a result of the above mentioned, it was evidenced that at the beginning of the evaluated period the inventory management was deficient, which improved progressively with the application of new policies generating a positive effect on profitability. The research concludes that according to the analysis carried out, the company has the tools to develop the activities correctly, a fact that generates improvement in the management processes and that the implementation of the new policies has had a satisfactory influence on profitability, generating a ROS of $4.75 \%$, ROA of $4.48 \%$, ROE of $37 \%$ and ROCE of $112 \%$.
\end{abstract}

Keywords: Management. Inventory. Policies. Profitability. 


\begin{abstract}
O objetivo da pesquisa era determinar o efeito da gestão de estoques na rentabilidade da empresa automotiva MOTORS SHOW SAC no distrito de Tarapoto, Província e Departamento de San Martin, Perú. Este estudo levou em conta artigos científicos orientados e publicados na EBSCO HOST, redalyc, ProQuest, SciElo, Scopus, Dialnet e SciencieDirect com base nos últimos 5 anos. Metodologia: É aplicada porque se baseou em contribuições teóricas para soluções alternativas, com uma abordagem quantitativa, escopo descritivo, projeto transversal e não experimental porque nenhuma variável foi manipulada. Como resultado do acima mencionado, ficou evidente que, no início do período avaliado, a gestão de estoques era deficiente, o que melhorou progressivamente com a aplicação de novas políticas, gerando um efeito positivo sobre a rentabilidade. A pesquisa conclui que, segundo a análise realizada, a empresa dispõe de ferramentas para desenvolver as atividades corretamente, fato que gera melhorias nos processos de gestão e que a implementação das novas políticas teve uma influência satisfatória na rentabilidade, gerando um ROS de 4,75\%, ROA de $4,48 \%$, ROE de $37 \%$ e ROCE de $112 \%$.
\end{abstract}

Palavras-chave: Administração. Estoques. Políticas. Rentabilidade.

\title{
1. INTRODUCCIÓN
}

En las empresas la gestión de inventarios es importante porque brinda soporte a los procesos logísticos que se encuentran inmersas en ellas el mismo que permitirá el desarrollo continuo de sus operaciones en el mercado. Según Juca et al. (2019) la gestión de inventarios es un pilar fundamental para el desarrollo de sus actividades porque se convierte en una fuente de ingresos cuando se lleva la rotación de las mercaderías, es por ello que al contar con un inventario óptimo satisfacen las necesidades del cliente final.

En el plano internacional, como es el caso de Colombia, la gran mayoría de empresas que prestan servicios están obligadas a realizar una gestión de inventarios totalmente eficiente con el propósito de lograr ser más competitivas, esto implica tener a disponibilidad los elementos necesarios (repuestos, insumos, productos en proceso, materiales terminados, etc.) y garantizar los mismos en un lugar adecuado y en condiciones perfectas (Salas et al., 2016).

En el Perú, el sector automotriz registró en Julio del 2021 un crecimiento del $12.13 \%$ en la adquisición de vehículos en comparación al año anterior (durante pandemia). Sin embargo; frente a la incertidumbre política y el factor como el alza del tipo de cambio viene afectando a los precios de las unidades, hecho que resta dicha recuperación que se venía viendo. Así mismo este crecimiento ha tenido impacto en los inventarios de las empresas con la importación de los suministros (lubricantes, partes de motor, filtros, neumáticos, partes eléctricas, partes de carrocería, baterías, entre otros) con un 50\% relacionado con el año precedente, considerando que la rentabilidad de las empresas mejoraría en el corto plazo (Asociación Automotriz del Perú - AAP, 2021).

La empresa en estudio es un concesionario autorizado que se desempeña en el rubro automotriz, mencionan que en ejercicios anteriores se han suscitado irregularidades en la gestión de sus inventarios, producto de los malos manejos por el personal e incluso por deficiencias en programas sistemáticos que a su vez han impedido un control correcto de las mercaderías, generando inestabilidad económica al cierre de año con cifras en la rentabilidad por debajo de lo planificado.

Según Ortega et al. (2017) como otro factor tenemos al mal manejo en su evaluación debido a su materialidad como activo de la empresa, puesto que distorsionaría cifras en los estados financieros de la empresa ocasionando serios problemas económicos. Por ende, es esencial mantener un monitoreo y vigilancia cuidadosa a la gestión de inventarios. Las diferentes causas que generan problemas en la gestión de los inventarios son debido a las reiteradas desorganizaciones y carencias de instrumentos de control sobre los inventarios con los que cuenta la empresa, tanto así que ocasiona el uso inadecuado de los recursos, generando perjuicio económico a la empresa (Angulo, 2019). 
De mantenerse este problema y no tener alternativa de solución, la empresa no se encontraría al nivel con otras organizaciones del mismo sector cayendo en adquisiciones innecesarias de productos que no tiene rotación y las consecuencias incidieran negativamente en sus ingresos, rentabilidad y beneficios (Cabrera et al. 2019).

De esta manera se formula la pregunta general de investigación ¿Cuál es el efecto de la gestión de inventarios en la rentabilidad de la empresa Motorsshow Tarapoto del distrito de Tarapoto? y los específicos ¿cuál es la gestión de inventarios de la empresa Motorsshow Tarapoto del distrito de Tarapoto? ¿cuál es la rentabilidad de la empresa Motorsshow Tarapoto del distrito de Tarapoto

Así mismo, se presentó como objetivo general que es determinar el efecto de la gestión de inventarios en la rentabilidad de la empresa Motorsshow Tarapoto del distrito de Tarapoto. Como objetivos específicos: analizar la gestión de inventarios de la empresa Motorsshow Tarapoto del distrito de Tarapoto y evaluar la rentabilidad de la empresa Motorsshow Tarapoto del distrito de Tarapoto, los periodos de evaluación son desde el ejercicio 2017 al 2020.

La investigación se justificó por conveniencia según Hernández-Sampieri y Mendoza (2018) el cual permitió conocer los beneficios al realizar el estudio a la empresa y el tener en cuenta las acciones correctivas para solucionar el problema. Así mismo se justifica por su relevancia social porque tiene el fin de contrarrestar los riesgos en relación a la gestión de inventarios mejorando la rentabilidad de la entidad.

En la investigación se determinó como objetivo general: determinar el efecto de la gestión de inventarios en la empresa Motorsshow Tarapoto del distrito de Tarapoto y los específicos: analizar la gestión de inventarios de la empresa Motorsshow Tarapoto del distrito de Tarapoto; así también evaluar la rentabilidad de la empresa Motorsshow Tarapoto del distrito de Tarapoto.

Como hipótesis general se presentó que el efecto de la gestión de inventarios en la rentabilidad de la empresa Motorsshow Tarapoto del distrito de Tarapoto.

En el fundamento teórico mencionaremos los estudios relacionados al tema de investigación, así como teorías similares a ésta que sustenta el uso de las variables. Por otra parte, en la metodología se mencionará el tipo y diseño de la investigación, de igual manera las técnicas e instrumentos empleados. En los resultados y discusión se conocerá las similitudes con otras investigaciones. Finalmente, las conclusiones y recomendaciones sobre el desarrollo de la investigación.

\section{FUNDAMENTO TEÓRICO}

Asencio et al. (2017), en su artículo desarrollado en Ecuador, teniendo como objetivo examinar el inventario en una distribuidora farmacéutica con el fin de precisar como incide en la rentabilidad. Se desarrolló una investigación exploratoria y descriptiva, teniendo una población de 25 trabajadores que laboran en diferentes áreas de la distribuidora obteniendo una muestra de quince empleados que tienen relación directa con el área administrativa y contable. Concluyeron que la gestión del inventario que se desarrolló en el 2012 incide negativamente en la rentabilidad de tal modo que generó pérdidas en ese ejercicio.

expresa que la empresa en estudio carece de control de los inventarios y que en último año afectó de manera negativa en la rentabilidad generando una pérdida de \$1'030,056 por los malos manejos.

El aporte de Garrido y Cejas (2017), en su artículo desarrollado en Ecuador, planteó como objetivo analizar la eficiencia del inventario aplicando modelos estadísticos matemáticos con el fin de conocer los costos inherentes de los productos que son vendidos por las PYMES. Como muestra tuvo a 3 empresas, como metodología empleó modelos matemáticos teniendo como resultado que 
los principales problemas de una gestión de inventarios se encuentran en el manejo y en el abastecimiento de los productos, concluyendo que los inventarios tienen un impacto significativo en todas las etapas de la administración que son los componentes de un sistema de gestión adecuado y confiable para las PYMES.

En el artículo realizado por Prempeh (2016), planteó como objetivo analizar la gestión de inventarios y el impacto que este genera en la rentabilidad de las empresas manufactureras en Ghana, su estudio fue transversal, donde se analizó informes financieros anuales de lo recopilado en los años 2004 a 2014 sobre cuatro organizaciones manufactureras que cotizan en GSE la Bolsa de Valores de Ghana, su muestra fue la misma población, aplicó la correlación de Pearson donde concluye que la gestión de inventarios tiene una fuerte relación positiva en la rentabilidad de las empresas manufactureras; es decir, el 1\% de una eficiente gestión de inventarios ocasiona un 9.35\% de impacto positivo en la rentabilidad de la industria manufacturera.

Un estudio en Lima, autor que su objetivo fue presentar a una empresa automotriz una nueva alternativa con respecto gestión de inventarios con el fin tener a disponibilidad repuestos y piezas de vehículos, la investigación fue descriptivo y cuantitativo, se recolectó información del área de compras para realizar el programa piloto donde se evaluó desde el inicio del proceso de compras hasta realizar la venta al cliente final dando resultados alentadores, su estudio resultó eficiente en la áreas de la empresa beneficiando a la gestión de inventarios convirtiendo al flujo de ingresos en constante, el autor concluye que implementar este modelo de gestión de inventarios logró un aumento del $7 \%$ en la rentabilidad, es decir el aporte produce un efecto positivo en la empresa (Rodríguez et al., 2020).

En Trujillo se encontró el artículo de Pastor y Javez (2017) quien desarrolló una propuesta de modelo de inventario para perfeccionar el ciclo logístico en una sucursal de la empresa en estudio, empleó un método experimental manipulando la variable gestión de inventarios con el propósito de conocer el efecto que genera en los costos logísticos, concluyendo que el método Winter era el adecuado debido que generó beneficios económicos en base a la ventas incrementando en un 10\% para el 2017 en comparación con el 2016 y porque se tuvo un ahorro notable de los costos logísticos en un $41.14 \%$.

Entre las dimensiones de la gestión de inventarios que propone Norazira et al. (2017), se tiene: procesos, que es la acción de seguimiento de las entradas, almacenamiento y despacho de mercaderías (Pacheco, 2019); control, consiste en la revisión minuciosa del procedimiento y manejo de mercaderías que posee el almacén de una empresa, así mismo la rotación en un periodo exacto (Tenesaca \& Maji, 2018); políticas, son estrategias planteadas por la directiva de una empresa, desde el propietario hasta el responsable del cuidado del almacén, con el fin de hacer un uso correcto de los recursos (León et al., 2020).

En cuanto a las dimensiones de la segunda variable que es la rentabilidad tenemos el aporte de Vinayagamoorthi et al. (2015), Rentabilidad de las ventas (ROS), beneficio que se obtiene de los ingresos de la empresa o conocido también como el margen operativo de la empresa (Hyblova \& Skalicky, 2018); (ROA) rentabilidad sobre activos, mide la eficiencia y eficacia de los activos para que genere ingresos a la empresa (Hossain, 2020) mientras mayor sea el resultado del rendimiento de los activos, más positiva será la rentabilidad (Lesáková et al., 2019) ; (ROE) Rendimiento sobre recursos propios, es la ganancia que se genera a partir de la inversión utilizando el capital (Pointer \& Dinh, 2019); (ROCE) rendimiento del capital empleado, indicador que mide los ingresos operativos de la empresa en base al capital invertido (Cyril \& Singla, 2020).

\section{PROCEDIMIENTOS METODOLOGICOS}

\section{Tipo de investigación}


Según CONCYTEC (2019) la presente investigación fue tipo aplicada porque para conocer el efecto de la gestión de inventarios en la rentabilidad se dependió de aportes teóricos, que fueron base de alternativas de solución.

\section{Diseño de investigación}

La investigación es de enfoque cuantitativo, de corte transversal, diseño no experimental y con alcance descriptivo debido a que no se manipuló ninguna variable, puesto que el objetivo de estudio es determinar el efecto de la gestión de inventarios en la rentabilidad de una empresa automotriz del distrito de Tarapoto, ejercicios 2017-2020.

\subsection{Presentación del diseño de la investigación}

En este apartado se muestra la figura de la relación entre ambas variables, es decir nos da conocer el efecto que tiene la gestión de inventarios en la rentabilidad, que se expresa de la siguiente manera:

Donde:

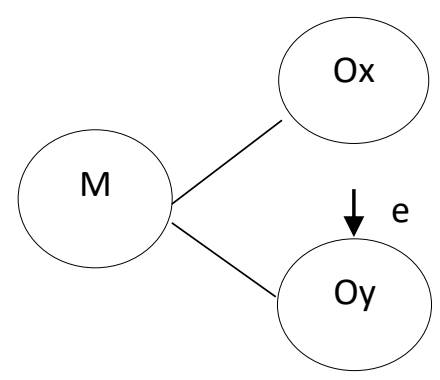

$\mathrm{M}=$ Empresa automotriz

$\mathrm{Ox}=$ Gestión de inventarios

$\mathrm{Oy}=$ Rentabilidad

$\mathrm{e}=$ Efecto

\section{Variable 1: Gestión de Inventarios}

Conjunto de procedimientos, controles y políticas que supervisan sistemáticamente el nivel de los inventarios en un determinado momento (Norazira et al., 2017).

\section{Variable 2: Rentabilidad}

Indicador que mide el nivel de efectividad del rendimiento de una empresa en sus operaciones, la utilidad que es calculada después del impuesto expresa el rendimiento de esas operaciones (Yuliani et al., 2020).

\section{Técnicas de recolección de datos}


Para la presente investigación se empleó como técnica la entrevista y el análisis documental para la gestión de inventarios lo cual se aplicó al área logístico de una empresa automotriz.

Para la variable rentabilidad se utilizó la técnica de análisis documental con lo que se analizó los estados financieros de los ejercicios 2017-2020.

\section{Instrumentos de recolección de datos}

Se empleó la guía de entrevista y ficha de registro como instrumento con respecto a la gestión de inventarios. Mientras que la ficha de registro se utilizó para la rentabilidad, ambos instrumentos fueron validados por juicio de 03 expertos.

\section{Procedimientos}

Se contactó y coordinó con la gerencia de la empresa para tener acceso y consentimiento respectivo a la información de los ejercicios 2017-2020 y así realizar la ejecución de la investigación.

En cuanto a la primera variable procedió a realizar las entrevistas a dos personas que son parte de personal de la empresa y que están vinculadas al área de almacén.

De la misma manera para la segunda variable el área contable nos facilitó los estados financieros de los años en estudio para realizar el análisis documental respectivo; de tal modo que se procesó para analizar los resultados, ayudando así a responder los objetivos planteados inicialmente.

\section{Método de análisis de datos}

Para la investigación se empleó como método de análisis el descriptivo desarrollándose de la siguiente manera:

Se recolectó información de manera independente para cada variable, una vez que el personal brindó las entrevistas se revisó y procesó toda la información para la variable gestión de inventarios.

En cuanto a la segunda variable rentabilidad se analizó los estados financieros para la posterior elaboración de las ratios ROS, ROA, ROE, ROCE donde se vio el comportamiento y efecto generado en los ejercicios 2017-2020.

\section{RESULTADOS Y DISCUSIÓN RESULTADOS}

De acuerdo a lo recopilado se procedió a realizar el análisis respetando el orden de los objetivos, el mismo que tiene el propósito de dar respuestas a las preguntas de investigación.

Objetivo específico 1: Analizar la gestión de inventarios de la empresa automotriz MOTORS SHOW SAC del distrito de Tarapoto, Provincia y Departamento de San Martin, Perú. 
Tabla 1. Rotación del inventario de la empresa automotriz MOTORS SHOW SAC del distrito de Tarapoto, Provincia y Departamento de San Martin, Perú.

\section{Indicador/Ejercicio}

Rotación de Inventario ${ }^{\text {a }}$

Rotación de Inventario en

días
2017

5

73
2018

8

5

\section{Información obtenida de los estados financieros}

\section{Interpretación:}

Se percibe en la siguiente tabla como la empresa automotriz del distrito de Tarapoto aprovecha sus inventarios, en el año 2017 tuvo una rotación de 5 veces realizándose la venta en 73 días, de acuerdo al sector en el que se encuentra lo usual es entre 4 a 5, logrando ser un índice bajo pero aceptable; sin embargo en dicho ejercicio ocurría irregularidades en la gestión de inventarios generando el ROA más bajo en ese entonces del 3\%, mientras que en el ejercicio 2018 se logra incrementar 3 puntos donde logrando una rotación en 45 días.

En el ejercicio 2019 la empresa implementó nuevas políticas logrando facilitar progresivamente la gestión de los inventarios; sin embargo, en la tabla podemos observar una caída de 3 veces en dicho año en comparación al anterior siendo las ventas cada 73 días nuevamente, es decir la inmovilización del stock se volvió permanente, la empresa tuvo cifras exorbitantes en su inventario, con el propósito de contar con ellos de manera inmediata.

Tabla 2. Acciones vinculadas a la gestión de inventarios según personal de la empresa automotriz del distrito de Tarapoto

\begin{tabular}{ll}
\hline Indicador & \multicolumn{1}{c}{ Acciones ${ }^{\text {a }}$} \\
\hline $\begin{array}{l}\text { Comprobación documentaria de entrada de } \\
\text { mercaderías }\end{array}$ & $\begin{array}{l}\text { El proceso de la recepción es con una guía de } \\
\text { remisión, posterior a ello se procede con la } \\
\text { identificación de la factura de compra electrónica, } \\
\text { registro en el sistema, verificación de la cantidad de } \\
\text { mercadería e ingreso al almacén. }\end{array}$ \\
\hline Ubicación de mercaderías & $\begin{array}{l}\text { Las mercaderías se encuentran ordenados en estantes } \\
\text { de metal y fueron de acuerdo al tipo de mercadería } \\
\text { (lubricantes, piezas, filtros, pastillas, aditivos, etc.), } \\
\text { pedidos y cancelados. }\end{array}$ \\
\hline
\end{tabular}

El despacho de las mercaderías se efectúa cuando se realiza el mantenimiento a vehículos y por venta directa de mostrador.

Autorización del despacho de mercaderías

Sustentan el despacho el vale de salida de almacén interno, boleta y factura de venta. 
Sistema de actualización de Mercaderías

Se ha estandarizado con el pasar de los años, sin embargo, no permite tener un control real de las mercaderías y más aún al no realizar los inventarios con continuidad.

Los productos están codificados desde fábrica y con ciertos productos el sistema genera un código de barras.

Codificación de mercaderías

Acceden a las mercaderías según marca y modelo de vehículo.

Toma de inventario físico

La toma de inventario es de forma descontinuada, en diferentes periodos y cuando consideran necesario. Así mismo emiten reportes sobre la cantidad de mercaderías cuando la alta gerencia lo solicite.

Reporte de riesgos internos de almacén

La empresa no cuenta con un plan de contingencia; sin embargo, ya están en el proceso de elaboración y emiten un informe cuando gerencia lo solicita, cada 2 meses.

Nota. ${ }^{a}$ Datos obtenidos a partir de la entrevista realizada al asesor de repuestos y almacenero

\section{Interpretación:}

De acuerdo a la información brindada por el personal de la empresa automotriz del distrito de Tarapoto se evidencia como resultado que hoy en día los procesos se efectúan correctamente, las nuevas políticas de gestión han permitido la progresiva mejora en recepción de los productos, el acceso a la ubicación y el despacho de los mismos de acuerdo a los parámetros establecidos.

Así mismo el personal no revisó oportunamente ciertos productos de manera continúa generando una pérdida por la inversión realizada e ingresos adicionales por un total de S/ 29,220.00 haciendo caer a la empresa en adquisiciones excesivas de productos que al mismo tiempo volvió a la rotación de los mimos en lenta tal como se muestra en la tabla 1 donde la venta se realizaba cada 73 días al año.

En el ejercicio 2020 a pesar de haber sido un año atípico por el COVID-19 fue el de mayor impacto por la cantidad de ventas realizadas, la empresa utilizó estrategias de ventas para dar salida a los productos logrando hacerlo en 24 días al año, a mayor rotación menos días de almacenamiento. De no haber sido por las pérdidas monetarias la empresa habría obtenido mayores ingresos y por ende mayor porcentaje de utilidad.

Tabla 3 Lista de productos en mal estado

\begin{tabular}{|c|c|c|c|c|c|c|}
\hline Cod. & Cod. Bar. & Descripción & $\begin{array}{c}\text { Unid } \\
\text { ad }\end{array}$ & $\begin{array}{c}\text { Cantida } \\
\text { d }\end{array}$ & $\begin{array}{l}\text { Costo } \\
\text { Unitario }\end{array}$ & Costo Total \\
\hline 10918 & 7700107555 & Taquies & Und & 16.00 & 199.64 & $3,194.24$ \\
\hline 11411 & 0947112151000 & $\begin{array}{l}\text { Ampolleta foco } 12 \mathrm{v} \\
60 \mathrm{w}\end{array}$ & Und & 20.00 & 134.75 & $2,695.01$ \\
\hline 10006 & 7750373010795 & $\begin{array}{l}\text { Limpiador de frenos } \\
500 \mathrm{ml}\end{array}$ & Und & 50.00 & 11.60 & 580.03 \\
\hline 11748 & 497206897R & Tubo De Alt Pre & Und & 1.00 & $2,200.62$ & $2,200.62$ \\
\hline
\end{tabular}




\begin{tabular}{|c|c|c|c|c|c|c|}
\hline 10849 & KA1C57K30A & Unidad S.A.S & Und & 1.00 & $2,174.66$ & $2,174.66$ \\
\hline 10595 & $9520077 \mathrm{~GB} 200$ & Compresor A/C & Und & 1.00 & $2,133.86$ & $2,133.86$ \\
\hline 10341 & U20515179 & Tubo Salida Agua & Und & 4.00 & 530.12 & $2,120.48$ \\
\hline 11568 & QONT146673 & Espejo Retrovisor & Und & 2.00 & 977.39 & $1,954.78$ \\
\hline 10170 & DM0R63900 & Parabrisas Delt & Und & 1.00 & $1,780.48$ & $1,780.48$ \\
\hline 11337 & 43210M83P202 & $\begin{array}{l}\text { Llanta Aluminio } \\
\text { (T3/4ptas) }\end{array}$ & Und & 1.00 & $1,028.01$ & $1,028.01$ \\
\hline 11685 & 310216010 & $\begin{array}{l}\text { Sae } 50 \text { Gulf Ht Fluid } \\
\text { T0-4 }\end{array}$ & B1 & 4.00 & 253.80 & $1,015.20$ \\
\hline 10826 & 7891799195342 & $\begin{array}{l}\text { Hsw } 300 \text { Plus } \\
\text { Limpiador De } \\
\text { Sistemas De Aire }\end{array}$ & Und & 20.00 & 60.00 & $1,200.00$ \\
\hline 10846 & AK-C01 & $\begin{array}{l}\text { Cámara De Retroceso } \\
\text { Akron }\end{array}$ & Und & 3.00 & 105.00 & 315.00 \\
\hline 10820 & 98618375 & $\begin{array}{l}\text { Mag } 1 \text { Sellador De } \\
\text { Neumáticos }\end{array}$ & Und & 10.00 & 28.33 & 283.30 \\
\hline 10823 & 5861111300 & $\begin{array}{l}\text { Aditivo Limpiador De } \\
\text { Inyectores Wurth }\end{array}$ & Und & 20.00 & 20.88 & 417.60 \\
\hline 10824 & 5861103300 & $\begin{array}{l}\text { Aditivo Para Gasolina } \\
\text { Wurth }\end{array}$ & Und & 20.00 & 18.65 & 373.00 \\
\hline 10825 & 5861501300 & $\begin{array}{l}\text { Aditivo Sellador Para } \\
\text { Radiador Wurth }\end{array}$ & Und & 20.00 & 28.33 & 566.60 \\
\hline 11436 & 4050641543882 & $\begin{array}{l}\text { Foco Lagrimón } \\
\text { Mediano Wurth }\end{array}$ & Und & 30.00 & 6.90 & 207.00 \\
\hline 10361 & 1D0143980AOT & $\begin{array}{l}\text { Flexible Freno } \\
\text { Delantero Derecho }\end{array}$ & Und & 12.00 & 0.63 & 7.51 \\
\hline 12110 & 300018 & Tapón M-18 X1.5 & Und & 1.00 & 7.35 & 7.35 \\
\hline 10898 & TYCO11862RL & $\begin{array}{l}\text { Conector De Cinturón } \\
\text { De Seguridad }\end{array}$ & Und & 2.00 & 3.50 & 7.00 \\
\hline 11427 & 5208010 & $\begin{array}{l}\text { Fusible Wurth 10a } \\
\text { Largo }\end{array}$ & Und & 18.00 & 0.35 & 6.30 \\
\hline 11428 & 5208005 & $\begin{array}{l}\text { Fusible Wurth 5a } \\
\text { Largo }\end{array}$ & Und & 20.00 & 0.30 & 6.00 \\
\hline 10157 & 89059061 & $\begin{array}{l}\text { Foco Narva De } 2 \\
\text { Contactos }\end{array}$ & Und & 2.00 & 3.00 & 6.00 \\
\hline 11071 & 7703097181 & (Pe) Ampolleta & Und & 1.00 & 5.81 & 5.81 \\
\hline 10901 & B2011152600 & $\begin{array}{l}\text { Soporte Manilla Int } \\
\text { Puerta Tras Der }\end{array}$ & Und & 1.00 & 5.67 & 5.67 \\
\hline 11425 & 5208020 & $\begin{array}{l}\text { Fusible Wurth 20a } \\
\text { Largo }\end{array}$ & Und & 18.00 & 0.30 & 5.40 \\
\hline 11429 & 520830030 & $\begin{array}{l}\text { Fusible Wurth 30a } \\
\text { Corto }\end{array}$ & Und & 18.00 & 0.30 & 5.40 \\
\hline 11434 & 520830005 & $\begin{array}{l}\text { Fusible Wurth 5a } \\
\text { Corto }\end{array}$ & Und & 18.00 & 0.30 & 5.40 \\
\hline 11424 & 5964035 & $\begin{array}{l}\text { Fusible Wurth D25 A } \\
\text { Largo }\end{array}$ & Und & 16.00 & 0.32 & 5.12 \\
\hline
\end{tabular}




\begin{tabular}{|c|c|c|c|c|c|c|}
\hline 11423 & 5208030 & Fusibles Wurth Largo & Und & 16.00 & 0.30 & 4.80 \\
\hline 11426 & 5208015 & $\begin{array}{l}\text { Fusible Wurth 15a } \\
\text { Largo }\end{array}$ & Und & 15.00 & 0.30 & 4.50 \\
\hline 11662 & 43281M67L0000 & Tuerca Rin Rueda & Und & 1.00 & 4.29 & 4.29 \\
\hline 11430 & 520830025 & $\begin{array}{l}\text { Fusible Wurth 25a } \\
\text { Corto }\end{array}$ & Und & 14.00 & 0.30 & 4.20 \\
\hline 11969 & 1987529034 & Mini Fusible 30 Amp & Und & 3.00 & 1.00 & 3.00 \\
\hline 11431 & 520830020 & $\begin{array}{l}\text { Fusible Wurth 20a } \\
\text { Corto }\end{array}$ & Und & 8.00 & 0.35 & 2.80 \\
\hline 11415 & MS000009 & Foco Lagrima & Und & 1.00 & 2.50 & 2.50 \\
\hline 11777 & 44329190 & Pernos M-14 X 25 & Und & 1.00 & 2.50 & 2.50 \\
\hline 11422 & MS000008 & $\begin{array}{l}\text { Foco Lagrimón Doble } \\
\text { Contacto }\end{array}$ & Und & 1.00 & 2.50 & 2.50 \\
\hline 12688 & 81482768 & $\begin{array}{l}\text { Topes De Puerta } \\
\text { Chicos }\end{array}$ & Und & 2.00 & 1.00 & 2.00 \\
\hline 11432 & 520830015 & $\begin{array}{l}\text { Fusible Wurth 15a } \\
\text { Corto }\end{array}$ & Und & 5.00 & 0.35 & 1.75 \\
\hline 10884 & 015500820A000 & Perno 015500820a & Und & 2.00 & 0.72 & 1.44 \\
\hline 11661 & MS000021 & Diodos & Und & 2.00 & 0.50 & 1.00 \\
\hline \multicolumn{6}{|c|}{ Total Costo } & 24,350 \\
\hline \multicolumn{6}{|c|}{ Ganancia } & $20 \%$ \\
\hline \multicolumn{6}{|c|}{ Pérdida } & 29,220 \\
\hline
\end{tabular}

Nota: Und $=$ unidad, $\mathrm{Bl}=$ balde.

\section{Interpretación:}

La tabla da a conocer los productos dañados que se encontraban dentro del almacén al finalizar el ejercicio 2019, el personal no revisó oportunamente, así como también la empresa no realizaba inventarios de manera continua generando una pérdida por la inversión realizada e ingresos adicionales por un total de S/ 29,220.00 haciendo caer a la empresa en adquisiciones excesivas de productos que al mismo tiempo volvió a la rotación de los mimos en lenta tal como se muestra en la tabla 2 donde la venta se realizaba cada 73 días al año.

En el ejercicio 2020 a pesar de haber sido un año atípico por el COVID-19 fue el de mayor impacto por la cantidad de ventas realizadas, la empresa utilizó estrategias de ventas para dar salida a los productos logrando hacerlo en 24 días al año, a mayor rotación menos días de almacenamiento. De no haber sido por las pérdidas monetarias la empresa habría obtenido mayores ingresos y por ende mayor porcentaje de utilidad.

Objetivo específico 2: Evaluar la rentabilidad de la empresa automotriz MOTORS SHOW SAC del distrito de Tarapoto, Provincia y Departamento de San Martin, Perú.

Tabla 4. Análisis de ratios rentabilidad de la empresa automotriz MOTORS SHOW SAC del distrito de Tarapoto, Provincia y Departamento de San Martin, Perú. 


\begin{tabular}{ccccc}
\hline Indicador & $\mathbf{2 0 1 7}$ & $\mathbf{2 0 1 8}$ & $\mathbf{2 0 1 9}$ & $\mathbf{2 0 2 0}$ \\
\hline ROS & $1,91 \%$ & $2,33 \%$ & $2,30 \%$ & $4.75 \%$ \\
\hline ROA & $3 \%$ & $3,23 \%$ & $3,53 \%$ & $4.48 \%$ \\
\hline ROE & $16 \%$ & $19 \%$ & $18 \%$ & $37 \%$ \\
\hline ROCE & $36 \%$ & $43 \%$ & $51 \%$ & $112 \%$ \\
\hline
\end{tabular}

Nota $:$ ROS $=$ Rendimiento de las ventas, $\mathrm{ROA}=$ Rentabilidad sobre activos, $\mathrm{ROE}=$ Rentabilidad sobre recursos propios, $\mathrm{ROCE}=$ Rendimiento del capital empleado

\section{Información obtenida de los estados financieros}

En la tabla se muestra que la rentabilidad sobre activos se muestra que en el periodo 2017 obtuvo como resultado del $3 \%$ el índice menor a comparación de los otros ejercicios, todo ello debido a que hubo una menor rotación de los inventarios, siendo así que por cada S/ 1.00 invertido en el total de sus activos generó una ganancia de S/ 3.00, mientras que para el periodo 2018 incrementó un $0.23 \%$, obteniendo como resultado del $3.23 \%$ incrementando una mínima rentabilidad sobre el uso de los activos donde que por cada un S/ 1.00 generó como beneficio de S/ 3.23. En el ejercicio 2019 se implementa nuevas políticas, generando un incremento en la rentabilidad sobre los activos de $0.30 \%$ teniendo como resultado el 3.53\%, en el cual nos indica que por cada S/.1.00 en activos, tenemos un retorno en utilidad del S/ 3.53 y en el ejercicio 2020 el ROA se incrementa considerablemente en un $0.95 \%$, el cual tiene como resultado del $4.48 \%$ porque se dio una mayor rotación de los inventarios; es decir por cada S/. 1.00 invertido en sus activos, se generó como rentabilidad de $\mathrm{S} / 4.48$ y un nivel de ventas extraordinario.

La rentabilidad de las ventas en el ejercicio 2017 obtuvo un resultado del $1.91 \%$ es decir que por cada S/ 1.00 de ventas generó como utilidad el S/1,91. Para el año 2018 en comparación al periodo 2017, nos muestra como resultado el $2.33 \%$, pues aumentó el $0.43 \%$ esto quiere decir que por cada S/ 1.00 de ventas se ha tenido como beneficio S/ 2.33, en el ejercicio 2019 tal como se percibe en la tabla la empresa obtuvo un descenso del $0.03 \%$ debido a que la rotación de los productos se volvió lento, pero esto no indica que la empresa haya tenido pérdida, al contrario se obtuvo como resultado el 2.30\%; es decir que por cada $\mathrm{S} / 1.00$ de ventas ha tenido como rendimiento $\mathrm{S} / 2.30,0.03$ menos que el año anterior y por último en el ejercicio 2020 se obtuvo como resultado un crecimiento significativo con un índice de $4.75 \%$, beneficio que se obtuvo a raíz de una mayor rotación de inventarios y cantidad de ventas realizadas generando $\mathrm{S} / 4.75$ por cada $\mathrm{S} / 1.00$ de venta.

El rendimiento de capital empleado en el ejercicio 2017 fue de $36 \%$, no fue eficiente el uso del recurso de la empresa, por cada S/ 1.00 de capital empleado, la empresa generó S/ 36.00, para el ejercicio 2018 incrementa un 5\% de la rentabilidad de la empresa; es decir por cada S/ 1.00 de capital empleado, la empresa generó S/ 43.00, mientras que en el ejercicio 2019 aumenta un 8\%, generando como resultado del $51 \%$ y por último en año 2020 crece el margen considerablemente, dando a entender que tenemos un $112 \%$ por capital empleado en la empresa.

En cuanto a la rentabilidad sobre los recursos propios, podemos percibir en la tabla, que por cada S/ 1.00 que la empresa posee de capital propio obtuvo como utilidad $16 \%$ en el año 2017 , $19 \%$ en el $2018,18 \%$ en el año 2019 y el año 2020 el 37\% que tiene mayor aumento, índices favorables y superiores al ROA; es decir que la empresa tiene capacidad para generar utilidades con uso de sus fondos propios.

Objetivo General: Determinar el efecto de la gestión de inventarios en la rentabilidad de la empresa automotriz MOTORS SHOW SAC del distrito de Tarapoto, Provincia y Departamento de San Martin, Perú. 
La gestión de inventarios en el ejercicio 2017 no se realizó de manera correcta, gran parte de ello por la falta de control de las mercaderías; los procesos fueron desarrolladas de manera deficiente provocando que el inventario no rote con la rapidez que se planteó, dichas acciones se reflejan en los índices de rentabilidad obtenidos en dicho ejercicio. En el 2018 de acuerdo a la tabla 2 se percibe un ligero aumento en la rotación de mercaderías debido a la cantidad de ventas realizadas, generando una rentabilidad sobre las ventas (ROS) del $0.42 \%$ más que el año anterior.

En el ejercicio 2019, se implementa nuevas políticas mejorando progresivamente los procesos de la gestión y lo demuestra la tabla 5 donde el ROA crece $0.3 \%$ retornando en utilidad S/ 3.53 por cada s/ 1.00 invertido en nuestros activos. Sin embargo; al cierre de ese año se dió a conocer la lista de productos en mal estado generando pérdidas monetarias por S/ 29,220.00 debido a que la toma de inventarios no se ejecutaba periódicamente.

En el ejercicio 2020 la gestión de inventarios genera un impacto favorable a pesar de lo ocurrido, la misma se refleja en el rendimiento de las ventas con el porcentaje más alto de $4.75 \%$, es allí donde las estrategias de venta han jugado un papel importante dentro de la incertidumbre sanitaria, la mayoría de los clientes fueron beneficiados con el programa REACTIVA PERÚ, con la liberación de CTS, BONOS Y AFP causando en ellos seguridad para recurrir a nuestros servicios y a la compra de productos. Por lo tanto, todo ello se traduce a que la gestión de inventarios genera un efecto positivo en la rentabilidad de la empresa en estudio.

\section{DISCUSIÓN}

En la presente investigación se planteó como primer objetivo específico analizar la gestión de inventarios en la empresa automotriz del distrito de Tarapoto, según los aportes de Norazira et al. (2017), la gestión de inventarios es el conjunto de procedimientos, controles y políticas que supervisan. En los resultados del presente estudio se detectó, que durante el periodo del 2017 existía falencias, puesto que el control y manejo de las mercaderías no se realizaban correctamente, obteniendo así una rotación de 5 veces al año un índice bajo pero aceptable para el sector en el que se encuentra, a partir del 2018 se ve la mejora progresiva en la gestión de inventarios, en el ejercicio 2019 se aplica las políticas de inventarios en las cuales al cierre de dicho año sale a relucir una pérdida de S/ 29,220 por los productos en mal estado sistemáticamente el nivel de los inventarios en un determinado periodo. Estos resultados guardan similitud con el estudio realizado por Rodríguez et al. (2020) el cual indican que no existe una adecuada organización y gestión de inventarios por la rotura de stock de los repuestos que son utilizados en el servicio de post venta, el cual genera un 51\% de desatenciones; sin embargo, al aplicar el nuevo modelo de gestión mejora los servicios, la rotación de los repuestos y la reducción de las desatenciones en un $18 \%$.

En cuanto al segundo objetivo específico se consideró evaluar la rentabilidad en la empresa automotriz del distrito de Tarapoto. Según los aportes de Yuliani et al. (2020) menciona que es el indicador que mide el nivel de efectividad del rendimiento de una empresa en sus operaciones, la utilidad que es calculada después del impuesto expresa el rendimiento de esas operaciones. Entre los resultados de la investigación se obtuvo que los índices de rentabilidad sobre los activos (ROA) va en aumento desde un 3\% en el ejercicio 2017 a un $4.48 \%$ en el ejercicio 2020 fruto de las nuevas políticas en la gestión de inventarios; sin embargo, el rendimiento de las ventas (ROS) en el ejercicio 2019 sufre un descenso de $0.33 \%$ producto del incremento de los costos, así mismo la rentabilidad sobre los recursos propios (ROE) muestra una baja en el índice del 1\% obteniendo una utilidad del 18\% en comparación al año anterior; gracias al uso correcto y progresivo de la gestión de inventarios, la aplicación de nuevas políticas se ven reflejados con mayor impacto en el ejercicio 2020 donde se genera un ROS del 4.75\%, un ROA del 3.23\% y el eficiente uso de los recursos y capital de la empresa generando un ROE del 18\% y un ROCE del 51\%, aproximándose así a los objetivos planificados por la empresa. Los mismos guardan similitud con el estudio realizado por Valle y Valqui (2019), mencionan que la empresa del año 2016 al 2017 cuenta con un crecimiento 
favorable en la rentabilidad; es decir lograron generar utilidades, todo ello a raíz de realizar un adecuado manejo a sus inventarios, una proyección exitosa con respecto a la demanda de sus productos y así contar con un óptimo nivel de stock.

Como objetivo general, en la presente investigación se consideró determinar el efecto de la gestión de inventarios en la rentabilidad de la empresa automotriz del distrito de Tarapoto, según el aporte de Guevara (2019), para que una empresa genere altos índices de rentabilidad la gestión de inventarios tiene que ser correcta y eficiente, enfocando a la mejora de sus procesos y el óptimo uso de recursos con lo que cuenta y así lograr sus metas trazadas, incrementado el flujo de sus operaciones y su rentabilidad. Los resultados de la investigación coinciden con lo encontrado por Prempeh (2017), que en su artículo menciona que la gestión de inventarios impacta significativamente positiva en la rentabilidad de las empresas en Ghana, debido a que los fabricantes por considerar un factor importante y vital para el desempeño óptimo de sus producciones aplican adecuadamente la gestión de su inventario.

\section{CONCLUSIÓN}

Se concluye que el efecto de la gestión de inventarios en la rentabilidad de la empresa es positivo, de acuerdo al análisis realizado a la empresa muestra que desde la implementación de políticas la empresa cuenta con herramientas para desarrollar las actividades de manera eficiente, demostrando la progresiva mejora en los procesos de la gestión, hecho que incrementó los indicadores de rentabilidad siendo este último el más provechoso, todo ello representa el esfuerzo y compromiso de la empresa.

La empresa automotriz del distrito de Tarapoto muestra que la gestión de inventarios en la actualidad se desarrolla de manera correcta, debido a que la implementación de las nuevas políticas ha repercutido satisfactoriamente en el control de las mercaderías; sin embargo, es importante mencionar que en el 2019 su tuvo pérdidas monetarias producto de las mercaderías en mal estado el cual asciendo a S/ 29,220.00 repercutiendo en las ventas ya que se obtuvo ingresos por debajo de lo planificado pudiendo haber sido aún más beneficiosas.

Se concluye que la rentabilidad de la empresa automotriz del distrito de Tarapoto aumentó en los últimos ejercicios, el ROS creció a $4.75 \%$ en el 2020, el ROA a $4.48 \%$, el ROE a 37\% y ROCE a 112\% desde el 2017 al 2020 en comparación a los años anteriores. Dicho crecimiento se dio gracias a la cantidad de ventas realizadas generados por la adecuada gestión de inventarios en el último ejercicio.

Es necesario mencionar de manera puntual ciertas limitaciones en la investigación, uno de ellos es en cuanto a la selección de artículos porque se complementó con sectores multidisciplinarios debido a que se carecía del sector automotriz; la segunda fue la selección de artículos según del continente americano por el país de residencia de la empresa en estudio.

Finalmente, se recomienda a la gerencia de la empresa automotriz del distrito de Tarapoto, fomentar y fortalecer las políticas de inventarios debido a que es una herramienta fundamental en el desarrollo de actividades dentro del almacén, ello logrará que el personal tenga presente los lineamientos y fortalezca la comunicación en el manejo de los inventarios y para incrementar la rentabilidad, se recomienda a la gerencia implementar un plan logístico para llevar a cabo de una manera mucho más eficiente la gestión de inventarios y ello permita facilitar los procesos de recepción, custodia y despacho de mercaderías.

\section{REFERENCIAS}


Angulo, R. (2019). Control interno y gestión de inventarios de la empresa constructora Peter Contratistas S.R. Ltda. Gaceta Científica; Vol 5 Núm 2 (2019); 129-137. DOI: 10.46794/gacien.5.2.696

Asencio, C.; Gonzales, E. y Lozano, M. (2017). El inventario como determinante en la rentabilidad de las distribuidoras farmacéuticas. Revista de Ciencias de la Administración y Economía, vol. 7, núm. 13, 2017. Universidad Politécnica Salesiana, Ecuador. DOI: https://www.redalyc.org/articulo.oa?id=504551272009

Asociación Automotriz del Perú. (2021). Importación de Suministros 2021. https://aap.org.pe/estadisticas/importacion_suministros/importacion-de-suministros-2021/

Cabrera, R.; Vargas, J.; Shinno, M.; Maradiegue, F. y Álvarez, J. (2019). Design of a purchases and inventories management of a cleaning products distribute company [Diseño de una gestión de compras e inventarios de una empresa distribuidora de productos de limpieza]. DOI: DOI:10.18687/LACCEI2019.1.1.186

Guevara, I. (2019). Gestión de inventarios y su relación con La rentabilidad de la empresa check Avanzado Chiclayo e.i.r.l, 2018. (Tesis de grado, Universidad Señor de Sipán). Repositorio institucional https://repositorio.uss.edu.pe/handle/20.500.12802/5723

Garrido, I. \& Cejas, M. (2017). La gestión de inventario como factor estratégico en la administración de empresas. Revista científica eléctrica de Ciencias Gerenciales (13) 37, 109-129. DOI: https://www.proquest.com/docview/1967041595?accountid=37408

Hernández-Sampieri, R. \& Mendoza, C. (2018). Metodología de la investigación. Las rutas cuantitativa, cualitativa y mixta. Año de edición: 2018, ISBN: 978-1-4562-6096-5, 714 p.

Hyblova, E. \& Skalicky, R. (2018). Return on sales and wheat yields per hectare of European agricultural entities [Retorno de las ventas y rendimiento de trigo por hectárea de las entidades agrícolas europeas]. DOI: https://doi.org/10.17221/209/2017-AGRICECON

Hossain, T. (2020). Determinants of profitability: A study on manufacturing companies listed on the dhaka stock exchange [Determinantes de la rentabilidad: un estudio sobre las empresas manufactureras que cotizan en la bolsa de valores de dhaka]. DOI: 10.18488/journal.aefr.2020.1012.1496.1508

Juca, C.; Narváez, C.; Erazo, J. y Luna, K. (2019). Modelo de gestión y control de inventarios para la determinación de los niveles óptimos en la cadena de suministros de la empresa Modesto Casajoana Cía. Ltda. Digital Publisher CEIT, ISSN-e 2588-0705, (4) 3-1. DOI: https://dialnet.unirioja.es/servlet/articulo?codigo=7144054

León, K.; Moreno, V. y Díaz, J. (2020). El control de inventarios en el sector camaronero y su aporte en los estados financieros. https://dialnet.unirioja.es/servlet/articulo?codigo $=7608923$

Lesáková, L.; Ondrusová, A. y Vinczeová, M. (2019). Factors determining profitability of small and medium enterprises in selected industry of mechanical engineering in the Slovak republic - The empirical study [Factores que determinan la rentabilidad de las pequeñas y medianas empresas en industria seleccionada de mecánica ingeniería en la república eslovaca - El estudio empírico]. DOI: 10.15240/tul/001/2019-2-010

Norazira, A.; Anuar, N. y Ahmad, P. (2018), Inventory management effectiveness of a manufacturing companyMalaysian evidence [La eficacia de la gestión de inventario de una empresa de fabricación: pruebas de Malasia], International Journal of Law and Management, https://doi.org/10.1108/IJLMA-04-2017-0094

Ortega, A.; Padilla, S. y Ruiz, A. (2017). Nivel de importancia del control interno de los inventarios dentro del marco $\begin{array}{lllll}\text { conceptual } & \text { de } & \text { una } & \text { empresa. }\end{array}$ https://revistas.unisimon.edu.co/index.php/liderazgo/article/view/3261

Pacheco, D. (2019). Gestión de inventario en empresas distribuidoras de materia prima del sector panadero en el Estado Zulia. Revista de Investigación en Ciencias de la Administración http://doi.org/10.33996/revistaenfoques.v3i11.65 No. 11| Volumen 3 | Julio septiembre 2019. https://revistaenfoques.org/index.php/revistaenfoques/article/view/65 
Pastor, J. \& Javez, S. (2017). Modelo de inventario probabilístico con revisión periódica para mejorar la gestión del ciclo logístico de Lenmex Corporation S.A.C. DOI: dx.doi.org/10.18050/RevUcv-Scientia.v9n2a3

Pointer, L. \& Dinh, P. (2019). Predictors of Return on Assets and Return on Equity for Banking and Insurance Companies on Vietnam Stock Exchange [Predictores de rendimiento de activos y rendimiento de capital para empresas bancarias y de seguros en la bolsa de valores de Vietnam]. Entrepreneurial Business and Economics Review, 7(4), 185-198. DOI: https://doi.org/10.15678/EBER.2019.070411

Prempeh, K. (2015). The impact of efficient inventory management on profitability: evidence from selected manufacturing firms in Ghana [El impacto de la gestión eficiente del inventario en la rentabilidad: evidencia de la fabricación seleccionada Empresas en Ghana. International Journal of Finance and Accounting 2016, 5(1): 22-26. DOI: 10.5923/j.ijfa.20160501.03

Reglamento RENACYT. Artículo 5 Definiciones. Investigación Aplicada. https://portal.concytec.gob.pe/images/renacyt/reglamento_renacyt_version_final.pdf

Rodríguez, S.; López, H.; Viacava, G. y Del Carpio, C. (2020). Inventory planning and management in the automotive after-sales supply chain [Planificación y gestión de inventarios en la cadena de suministro del sector post venta automotriz]. Digital Object Identifier (DOI): http://dx.doi.org/10.18687/LACCEI2020.1.1.178 ISBN: 978958-52071-4-1 ISSN: 2414-6390

Salas K., Maiguel H. y Acevedo J. (2016). Metodología de Gestión de Inventarios para determinar los niveles de integración y colaboración en una cadena de suministro. Revista chilena de ingeniería, 25(2), 326-337. DOI: https://dx.doi.org/10.4067/S0718-33052017000200326

Tenesaca, M. \& Maji, E. (2018). Control de los inventarios y su incidencia en la rentabilidad para negocios que comercializan productos de primera necesidad. https://www.eumed.net/rev/oel/2018/07/control-inventarios rentabilidad.html

Valle, M. \& Valqui, A. (2019). Control de inventarios y su influencia en la rentabilidad de la empresa Armando Rodríguez. Tello - Representaciones Progreso 2016 -2017 (Tesis de grado, Universidad Nacional de San Martin)

Repositorio institucional http://repositorio.unsm.edu.pe/bitstream/handle/11458/3355/CONTABILIDAD\%20\%20RIOJA\%20\%20M\%c 3\%b3nica $\% 20$ Valle $\% 20$ Zuta $\% 20 \% 26 \% 20 \mathrm{Absal} \%$ c3\%b3n $\% 20$ Valqui $\% 20$ Trauco $\% 20$.pdf? sequence=1\&isAllo wed $=\mathrm{y}$

Vinayagamoorthi, V.; Murugesan, S. y Kasilingam, L. (2015). Impact of Firms' Profitability on Environmental Performance: Evidence from Companies in india [Impacto de la rentabilidad de las empresas en el desempeño ambiental: Evidencia de empresas en India]. DOI: 10.5901/mjss. 2015.v6n1p109

Yuliani, Y.; Suhartini, K.; Rasyid, U.; Samadi, B. y Robiyanto, R. (2020). Moderating effect of business environment to working capital and profitability in indonesia [Efecto moderador del entorno empresarial sobre el capital de trabajo y la rentabilidad en Indonesia]. DOI: 10.22201/fca.24488410e.2021.2480 